\title{
El abordaje de la cuestión juvenil y el desarrollo de sistemas de protección social y bienestar juvenil en gobiernos localles
}

\section{The youth issue approach and the social protection and youth welfare systems development in local governments}

\author{
Pereyra, Eduardo Javier
}

Eduardo Javier Pereyra Sobre el autor eduardojpereyra82@gmail.com CONICET, Argentina

PAPELES del Centro de Investigaciones de la Facultad de Ciencias Jurídicas y Sociales de la UNL Universidad Nacional del Litoral, Argentina

ISSN: $1853-2845$

ISSN-e: 2591-2852

Periodicidad: Semestral

vol. 12, núm. 23, 2021

papelesdelcentro@fcjs.unl.edu.ar

Recepción: 30 Agosto 2021

Aprobación: 15 Octubre 2021

URL: https://doi.org/10.14409/p.v12i23.10782

\section{(i)(2)}

Esta obra está bajo una Licencia Creative Commons AtribuciónNoComercial-CompartirIgual 4.0 Internacional.
Resumen: Los gobiernos locales despliegan un amplio conjunto de políticas juveniles y desarrollan distintos tipos de institucionalidad para el abordaje de la cuestión juvenil en sus comunidades. Este trabajo propone una clasificación categórica para la interpretación sistemática de las mismas: los sistemas de protección social y bienestar juvenil. Se analiza con un estudio cualitativo comparado sobre la potencia analítica de la teoría tipológica construida, mediante la recuperación histórica de las políticas juveniles diseñadas e implementadas en dos municipios de la provincia de Córdoba, Argentina. Además, se indaga sobre los factores que inciden en la conformación y desarrollo de cada uno de los sistemas de protección social y bienestar juvenil observados. La investigación realizada parte de la hipótesis de que éstos dependen de las características que adquieran los proyectos de gobierno a cargo de las gestiones locales, mediado por las capacidades estatales dispuestas al abordaje de lo juvenil y por la influencia que ejercen los actores extraestatales de la dinámica política local. El artículo expone los principales hallazgos a la luz del marco analítico propuesto.

Palabras clave: Cuestión Juvenil, Políticas Sociales, Sistemas de Protección Social, Gobiernos locales.

Abstract: Local governments deploy a broad set of youth policies and develop different types of institutions to address the youth issue in their communities. This work proposes a categorical classification for their systematic interpretation: social protection and youth welfare systems. It is analyzed with a comparative qualitative study on the analytical power of the typological theory built through the historical reconstruction of youth policies designed and implemented in two municipalities in the province of Córdoba, Argentina. In addition, it investigates the factors that affect the formation and development of social protection and youth welfare systems. The research carried out starts from the hypothesis that these depend on the characteristics acquired by the government projects in charge of local administrations, mediated by the state capacities willing to address the youth and by the influence exerted by extra-state actors on the dynamics local politics. The article presents the main findings from the proposed analytical framework. 
Keywords: youth Issue, social Policies, social Protection Systems, local Governments.

\section{Introducción}

En los municipios de Argentina existe una amplia heterogeneidad institucional de las políticas sociales juveniles. Para comprender de manera esquemática el conjunto de intervenciones que desarrollan los gobiernos locales para intervenir sobre la cuestión juvenil se propone la utilización de la categoría "sistemas de protección social y bienestar juvenil" y una clasificación de tres "tipos ideales" para dar cuenta de la diversidad sobre las producciones socio-estatales sobre lo juvenil que los Estados subnacionales realizan.

Además, se asume el desafío de analizar y dar cuenta de los factores que condicionan e influyen sobre la estructuración de los sistemas. El estudio propone que éstos se encuentran condicionados, principalmente, por los posicionamientos que asume un proyecto de gobierno frente a la cuestión social en general y la juvenil en particular y el efectivo compromiso que las distintas unidades estatales asumen en el diseño y ejecución de políticas juveniles mediante el despliegue de sus capacidades estatales y; la influencia que ejercen los actores de la dinámica política local.

Para avanzar en este sentido se usa el método comparativo en combinación con una estrategia cualitativa de análisis, que implica, para cada caso seleccionado, la reconstrucción del proceso -process-tracing (Nazareno, 2009)- del desarrollo histórico institucional de las políticas juveniles y de las estructuras del Estado a nivel local destinada a tales fines. Los casos concretos de análisis son los municipios de Villa María y Jesús María, de la Provincia de Córdoba, a lo largo del periodo 1999-2017.

Cabe aclarar que el análisis se centra en las políticas juveniles diseñadas e implementadas exclusivamente desde el nivel local, por lo que no se incluyen en el análisis los programas nacionales o provinciales ejecutados en los municipios. Además, se consideran para el estudio aquellas acciones que estén diseñadas pensando en el/la joven como sujeto de las políticas y que implique una problematización respecto a la cuestión juvenil (Scagliola, 2018).

El trabajo se organiza del siguiente modo. En el primer apartado se propone una definición de la categoría "sistema de protección social y bienestar juvenil y la designación de los valores que estos pueden asumir a partir de la construcción de una tipología. En el segundo apartado, se desarrolla el esquema interpretativo sobre los factores que explican la estructuración de los sistemas de protección juvenil, dando cuenta del marco conceptual utilizado para el análisis empírico. En el tercer punto se presenta el análisis de los tipos de sistemas observados en los municipios seleccionados como casos de estudios. El cuarto apartado expone de manera comparada las principales características que asumieron las variables explicativas en los casos estudiados, y finalmente, se cierra con los hallazgos y las conclusiones obtenidas. 


\section{Delimitaciones conceptuales: los sistemas de protección social juvenil y su clasificación tipológica}

La política social, y por ende la política juvenil, son un modo de definir y construir el bienestar de una sociedad, a la vez, que su proceso de diseño e implementación se encuentra políticamente mediado por las ideologías dominantes, los valores sociales hegemónicos, los conflictos de intereses entre actores, los dispositivos institucionales y los conocimientos y técnicas de actuación propios de cada sector (Fleury, 2000). Se afirma entonces, que las instituciones y las políticas de bienestar no son neutrales.

Para comprender las definiciones en torno a la cuestión juvenil y su abordaje estatal a nivel local se considera fundamental investigar las características que adoptan los sistemas de protección social y bienestar juvenil. ${ }^{1}$ Se los define como el conjunto de acciones públicas tendientes a proteger a las personas jóvenes de situaciones de exclusión social como las acciones tendientes a promover los derechos políticos, sociales y de construcción de ciudadanía juvenil (Cecchini, Filgueira y Robles, 2014; Balardini, 1999).

Para la definición de los tipos de protección, se consideran las posiciones frente a los instrumentos que generan acceso a la protección, niveles de cobertura, vías de financiamiento, grados de solidaridad y estratificación que generan y, las condiciones de ciudadanías generadas (Fleury, 2000).

A partir de las clasificaciones e interpretaciones sobre sistemas de protección y regímenes de bienestar realizados por Donati y Lucas (1987); Fleury (2000); Roca, Golbert y Lanari (2012) y Brugué y Gomá (1998) se propone la siguiente tipificación de modelos de protección social y de bienestar juvenil, para estudiar casos a nivel local: "Sistema Fragmentado Residual"; "Sistema Fragmentado Estratificante" y "Sistema Integrado".

El sistema "Fragmentado residual" se caracteriza por una intervención estatal que actúa ante las fallas mercantiles o imposibilidades de los jóvenes de canalizar sus necesidades a través del acompañamiento familiar. En este sentido, el o la joven debe presentar "pruebas" concretas de sus carencias y de los medios para hacer frente su situación y así habilitar los mecanismos que el Estado prevé para ayudar a las personas en estos casos específicos. Las políticas desplegadas son focalizadas en tanto "ayuda" a sectores vulnerables a palear alguna de sus necesidades o urgencias, pero sin abordar la problemática de fondo o estructurando medidas que permitan al joven incorporarse a trayectorias institucionales que le brinde herramientas, oportunidades y condiciones para "salir" de su situación de vulnerabilidad. En este sentido, la acción estatal tiene un sentido caritativo y de control social de la pobreza. Ante esto, los mecanismos y entramados institucionales desplegados por los gobiernos son muy poco complejos, de mirada cortoplacista, con una base normativa inexistente y un desarrollo organizativo muy escaso, pero de carácter burocratizado y con un alto valor hacia el territorio para "encontrar" beneficiarios y eficientizar así su intervención. Detrás de esta perspectiva hay una visión de ciudadanía invertida ya que se establece que el joven fracasó (individual y socialmente) en su trayectoria, con lo cual se vuelve objeto de la política.

En el sistema "Fragmentado estratificante" el Estado interviene en la cuestión juvenil para sostener y potenciar la inclusión de la persona joven en el 
sistema educativo y/o en la estructura productiva, con el objetivo de formarlo, acompañarlo y brindar oportunidades en su transición "regulada" a la vida de mundo adulto, ya que la juventud se define como una etapa de transición en la cual las y los jóvenes se ven atravesados por una serie de problemas "propios" de la edad pero también como consecuencia de la estructura socio-económica y productiva de las sociedades capitalistas. Se asienta en el modelo de inserción laboral juvenil basado en la "inversión en el capital humano" en el que las políticas son destinadas mayoritariamente a los "jóvenes integrados", funcionalizando "adecuadamente" la utilización del tiempo libre, y definiendo la juventud como "la esperanza del futuro" (Rodríguez, 2000). Se está en presencia entonces, de una ciudadanía regulada en tanto objetiviza y vincula al joven en su condición de estudiante o trabajador.

En el sistema "Integrado", el acceso a los beneficios está dado por la simple necesidad del joven en cuanto ciudadano de su localidad. No existe un "contrato" de condiciones y contraprestaciones para acceder a la política en la que el Estado invierte importantes recursos, generando un recurso mínimo vital para las juventudes que funciona como un mecanismo de redistribución hacia la población joven de carácter universalizable. No obstante, aún pueden implementarse políticas focalizadas hacia un sector específico de la población juvenil, pero la intervención aspira a llegar "universalmente" a todo este conjunto de potenciales beneficiarios. Cuanto menos segmentada sea la política menor será la consideración de la base territorial para su diseño e implementación, pero sí se desarrolla que un esquema normativo claro e institucionalizado para garantizar su intervención. La magnitud de la presencia e intervención estatal exige el despliegue de un sistema institucional amplio, complejo, plural, sólido e integrado. A su vez las políticas entienden a las y los jóvenes como sujetos de derecho, adoptando una perspectiva generacional en el diseño e implementación de las mismas (Rodríguez, 2003).

Finalmente, cabe señalar que estos modelos así presentados hacen referencia a "tipos ideales" en "estado puro", con lo cual cuando en una gestión coexistan claramente características de dos o más modelos, adquiere relevancia la clasificación de sistemas de protección juvenil "Híbridos”.

\section{Esquema interpretativo de los factores que inciden en la estructuración de los sistemas de protección juvenil}

Es innegable que existen grupos o actores con posiciones privilegiadas ante determinadas decisiones políticas o que poseen recursos para influir en la agenda y acciones de gobierno, pero también es indiscutible que el Estado posee sus propias lógicas independientemente de sus relaciones con la sociedad. Por ello, el esquema explicativo de los sistemas de protección juvenil adoptados por los municipios gira en torno a dos variables.

En primer lugar, el abordaje de la cuestión juvenil como resultado del "proyecto de gobierno" adoptado por la gestión, donde intereses, ideas y concepciones políticas de los funcionarios a cargo de la gestión cumplen un rol fundamental en su estructuración. Esto contribuye a explicar la formulación autónoma de políticas desde el aparato estatal. Además, se incluye el análisis de las "capacidades estatales" desplegadas por los gobiernos para la construcción 
de la institucionalidad destinada al abordaje de la cuestión juvenil. Un modo de analizar el efectivo compromiso asumido por las gestiones y analizar las intermediaciones existentes entre las decisiones adoptadas y los rasgos específicos que asumen las políticas juveniles.

Como segunda variable, atento a los potenciales factores externos, se incorpora en el análisis la influencia de los "actores en la dinámica política local" sobre los proyectos de gobierno y la institucionalidad que estos despliegan para el abordaje de la cuestión juvenil, es decir, los sistemas de protección social y bienestar juvenil.

\subsection{Proyectos de Gobierno}

Peter Hall introduce el concepto de policy paradigms, es decir marcos interpretativos que moldean objetivos y estrategias de los agentes estatales, como son el keynesianismo y el neoliberalismo, pero estos paradigmas están presentes tanto en políticos y burócratas como en el conjunto de la sociedad y operan de distintas maneras en unos y otros (Viguera, 2000).

Desde finales de la década de 1990, precisamente a partir del triunfo de Hugo Chávez en Venezuela en 1998, se viene señalando que los gobiernos nacionales de América Latina han realizado un "giro a la izquierda" (Stokes, 2008; Alegre, 2008), fundamentalmente si se los contrapone con los planes de ajuste estructurales-neoliberales del "Consenso de Washington" impuestos en el continente a partir de mediados de $1970 .^{2}$ No obstante, el posneoliberalismo no es antagónico al neoliberal en la medida que hasta el momento no constituyen un quiebre al sistema capitalista, pero cobija importantes rupturas y algunas continuidades con el mismo. Particularmente se distingue por la vuelta de la centralidad del Estado, la redistribución de la riqueza y la inclusión.

Sin embargo, a partir del "éxito" de diversos procesos destituyentes que forzaron mecanismos institucionales-constitucionales (que se han denominado como "Neogolpismos"3 o "Golpes Blandos"), se asistió en la región a un nuevo giro político, esta vez hacia la derecha. A esta nueva ola de derecha hay que agregar los triunfos electorales de Cartes en Paraguay (2013), Varela en Panamá (2014), Macri en Argentina (2015), el regreso de Piñera en Chile (2017) y Bolsonaro en Brasil (2018).

Esta discusión, indica que las categorías izquierda y derecha poseen vigencia teórica y empírica en América Latina (Lievesley y Ludlam, 2009) aunque las mismas se han reconfigurado y transformado respecto a sus categorizaciones clásicas previo a los años '80. En este trabajo se utiliza entonces, las categorías neoliberal y posneoliberal para definir los proyectos de gobierno (Ramírez Gallegos, 2012a; Sader, 2008; Stoessel, 2014).

\subsection{Actores de la dinámica política local}

Se entiende por actor a un sujeto individual o colectivo con capacidad de acción estratégica, es decir con capacidad de definir sus intereses, traducirlos en objetivos y diseñar una estrategia e implementarla mediante un curso de acción (Acuña, 2013). 
Se parte de entender a la elección de un tipo de sistema de protección y sus políticas como resultado de los procesos de inclusión/exclusión por los que se constituye la agenda social de gobierno y se definen y gestionan las políticas sociales. Para analizar a los actores y sus comportamientos es necesario comprender tres elementos: sus intereses, sus recursos y sus ideas/entendimiento. En función de esta perspectiva, se pone foco en las formas y contenidos de las interacciones de los actores que interviene en el ciclo de la política (Acuña, 2005).

En este último sentido, existe una perspectiva teórica que permite profundizar analítica y conceptualmente el rol de las ideas de los actores en la definición e implementación de las políticas: el marco de las coaliciones de causa o promotoras (advocacy coalition framework - $A C F$ - en su idioma de origen). De este enfoque, el estudio toma particularmente la existencia de un conjunto de actores al interior del propio subsistema político municipal, que influyen fundamentalmente sobre el sistema de creencias, visiones y representaciones respecto a las juventudes, la cuestión juvenil y por ende sobre las características asumidas por el municipio en torno al diseño e implementación de políticas públicas locales.

\subsection{Las capacidades estatales indicadas como indicador del efectivo compromiso de los proyectos de gobierno}

Según lo expuesto más arriba, las políticas públicas juveniles surgen desde los intereses y decisiones que un gobierno a cargo del poder ejecutivo defina y que, a su vez, pueden existir determinados actores que ejercen presiones para influir en las definiciones a cargo de los agentes estatales. No obstante, esto no significa que necesariamente lo que se defina en el plano político-ideológico-institucional sobre una agenda de gobierno y el tipo de políticas a diseñar e implementar, puedan ser llevadas a la práctica de manera directa. Es decir que existe una intermediación entre las decisiones adoptadas y las características concretas de la política que se ejecuta. Aquí aparece en juego otra dimensión, que es el de la capacidad estatal para desplegar e implementar programas (Viguera, 2000).

Incorporar las capacidades estatales permite valorar el grado de desarrollo e intensidad que en la realidad cotidiana de la gestión se llevan a la práctica esos sistemas de protección definidos política e ideológicamente por la relación entre actores estatales y sociales, funcionando como un indicador del compromiso efectivo de las gestiones en el desarrollo de políticas juveniles de acuerdo al tipo de proyecto de gobierno que se adopta.

La "capacidad indicada" es entendida como la eficacia administrativa del Estado para cumplir los objetivos establecidos a partir de la disponibilidad de determinados recursos humanos, materiales y tecnológicos (Oszlak, 2004).

\section{Los sistemas de protección y bienestar juvenil en los Estados locales}

El presente apartado aborda el desarrollo de políticas orientadas a la juventud, gestionadas por el Municipio de Jesús María y Villa María. Para ello, se realizó un recorrido histórico sobre cómo ingresó la cuestión juvenil en la agenda pública de la ciudad, las principales políticas juveniles diseñadas e implementadas, 
las estrategias de abordaje y su despliegue institucional a lo largo del periodo de estudio. En conjunto con la descripción de los programas y proyectos, se analizaron las miradas y posiciones de funcionarios y agentes estatales frente a la cuestión juvenil y el rol estatal en su abordaje. A través de entrevistas, documentos institucionales y notas periodísticas, se indagaron las definiciones y concepciones sobre las juventudes y, a su vez, cómo impactaron estas miradas en la estructuración de la respuesta estatal, para lograr una comprensión y caracterización del sistema de protección social y bienestar juvenil en función del marco conceptual propuesto.

\subsection{Jesús Maria (2003-2017): un sistema bibrido "fragmentado residual y estratificante"}

A partir de la indagación y análisis de la trayectoria histórica respecto a la institucionalidad municipal juvenil desde el año 2003 hasta finales del año $2017,{ }^{4}$ fue posible diferenciar dos grandes etapas en el diseño y gestión de políticas en la localidad de Jesús María, atravesada por una misma constante: la política pública juvenil anclada centralmente en un órgano gubernamental juvenil específico (OGJ). Dichas etapas se correspondieron con las gestiones de los dos intendentes que ocuparon el poder ejecutivo en dicho periodo, pertenecientes a un mismo partido de gobierno. La primera etapa concierne a las dos gestiones consecutivas ejercidas por el intendente Marcelino Gatica (2003-2007/2007-2011). En su transitar, a su vez, se lograron diferenciar dos momentos importantes: el de la génesis de la política juvenil y el de su posterior institucionalización. Dichos momentos concentran y contienen una serie de continuidades y rupturas que dan cuenta de tensiones y miradas contradictorias sobre el abordaje de lo juvenil.

La segunda etapa se corresponde con las dos gestiones del intendente Gabriel Frizza (2011-2015/2015-2017). Aquí también se identificaron dos momentos de gestión con características particulares. Durante el primer mandato se visualizó un fuerte retroceso y vaciamiento de la política juvenil, en un contexto de agudos conflictos políticos internos con el equipo de gestión del OGJ, que continuaba siendo el mismo que el de finales de la primera etapa. El segundo momento, se inició en el segundo mandato, donde se produjeron ciertos movimientos que insinuaron dar mayor rodaje al trabajo del Área de Juventud: cambio de equipo técnico y la propuesta de un Plan Joven para el municipio con nuevas líneas de acción.

A los fines de comprender el despliegue del sistema de protección y bienestar juvenil implementado desde 2003 a 2017 en la ciudad en términos esquemáticos, se resume sus principales características en el Cuadro 1. 


\begin{tabular}{|c|c|}
\hline & $\begin{array}{c}\text { Jesús María } \\
\text { Híbrido "Fragmentado residual v estratificante" }\end{array}$ \\
\hline Rasgos/Modalidad & Inclusión protectiva y productiva \\
\hline Principio & Caridad y solidaridad \\
\hline Enfoque sobre el bienestar & Enfoque de necesidades y Enfoque de capacidades \\
\hline Efecto & Estigmatización y estratificación \\
\hline Objeto y función de la intervención & Control social - Reproducción social \\
\hline Acceso a beneficios & Prueba de medios - Afiliación \\
\hline Tipos de beneficios & Transferencias no condicionadas \\
\hline Alcance de la política & Focalizadas \\
\hline Enfoque de la juventud & $\begin{array}{c}\text { Moralizantes - Juventud como problema } \\
\text { Juventud como etapa de transición. } \\
\text { Enfoque de derechos }\end{array}$ \\
\hline Ciudadanía juvenil & Invertida - regulada \\
\hline Valor del territorio & Intermedio \\
\hline Base normativa & Baja \\
\hline Modelo organizativo & Escaso \\
\hline Niveles de empowerment & Bajo - intermedio \\
\hline Desarrollo de modelo social local propio & Bajo \\
\hline
\end{tabular}

\section{Cuadro 1.}

Resumen de principales características del sistema de protección social y bienestar juvenil de Jesús María (2003-2017)

Elaboración propia en base a categorías de Donati y Lucas (1987); Fleury (2000); y Roca, Golbert y Lanari (2012), Brugué y Gomá (1998), Krauskopf (2000) y Rodríguez (2000 y 2003).

La política nació como programa para abordar centralmente a la juventud como una etapa de transición atravesadas por complejos riesgos y problemas, fundamentalmente respecto a las adicciones (de sustancias legales e ilegales) y la preocupación en torno a la sana ocupación del tiempo libre. ${ }^{5}$ Hay un objeto de control social por parte de la intervención estatal, cuya acción cobró sentido en la medida que el joven ha "caído" en problemas (principalmente por responsabilidad propia) y necesita cierto tutelaje para la corrección de su camino (idea de ciudadanía invertida). Al haber sido un dispositivo de prevención, principalmente de manera directa mediante la intervención clínica y el tratamien to ambulatorio, existió un escaso despliegue burocrático y normativo a los fines de dotar de institucionalidad a la política. Se desarrolló entonces una primera intervención de carácter residual y paliativa frente a una problemática puntual sin aspiraciones en desplegar un modelo de desarrollo local propio.

Esta primacía de paradigmas negativizantes sobre las personas jóvenes fue analizada críticamente por el intendente, cuestión que impulsó a modificar esta fuerte asociación de la juventud como etapa problema y en riesgo y avanzar sobre estrategias de formación y participación juvenil. Paulatinamente se fueron generando nuevos programas que incorporaron las ideas de una "juventud ciudadana", consolidando proyectos en torno a la participación juvenil. ${ }^{6}$ Como consecuencia, a fines del 2009 se decidió crear el Área de Juventud, conformando un nuevo equipo técnico a cargo de las políticas juveniles. Esto derivó, en la decisión política de la nueva coordinación de adoptar el enfoque de derechos como paradigma de trabajo. ${ }^{7}$ No obstante, el abordaje desde el modelo biopsicosocial continuó, pero anclado a un programa focalizado en el tratamiento ambulatorio de jóvenes con problemas de adicciones y talleres sobre drogodependencia dentro del área de salud (programa "jóvenes saludables"). 
Durante la denominada segunda etapa de gestión que abarcó las dos intendencias de Frizza (2011-2017) también se identificaron en su interior dos momentos: el de la primera gestión (2011-2015) marcado por la continuidad del equipo técnico de la etapa anterior en un contexto de altos niveles de conflictividad político-institucional con el poder ejecutivo. Desde el organismo se sostuvieron las mismas conceptualizaciones, paradigmas y enfoques respecto al abordaje de la cuestión juvenil, pero se detectó una importante disminución de la relevancia política del organismo para la gestión municipal, y como consecuencia, se dieron de baja a varias líneas de acción lo que derivó, hacia fines del mandato, en la renuncia del equipo y un cierre del organismo en la estructura municipal entre noviembre de 2015 y abril de 2016, coincidente con el inicio del segundo gobierno de Frizza. Por tales motivos a este periodo se lo denominó como el "momento de retroceso y vaciamiento" del Área de Juventud.

El segundo momento, tras el nombramiento de una nueva coordinación a cargo del Área, se dio paso a la reestructuración de la política juvenil en torno al Plan Jesús María Joven con la continuidad de los programas emblemas del organismo. No obstante, se detectaron algunas modificaciones en paradigmas y conceptualizaciones sobre lo juvenil, con un retorno del modelo biopsicosocial al organismo vertidas en el nuevo programa, eje de la intervención sobre la cuestión juvenil.

A lo largo de la trayectoria, esta convivencia de paradigmas en programas socioeducativos, sanitarios, de participación ciudadana generó que hubiese acciones que partieran de un principio de caridad y otras desde la solidaridad. En este mismo sentido, el enfoque de bienestar se asentó en el de necesidades (miradas sanitaristas) y el de capacidades (miradas de desarrollo de capital social) por lo cual el objeto y función de las intervenciones giraron alrededor del control social para los casos de abordaje de los factores de riesgo juvenil, el de la reproducción social para los de carácter educativo y los de realización del derecho de ciudadanía para los proyectos de participación juvenil.

Todas las políticas estuvieron focalizadas (con criterios más o menos amplios) a una población juvenil determinada, siendo los grandes sectores beneficiados las y los jóvenes estudiantes y en menor medida, jóvenes de sectores populares que específicamente se encontraban con problemas de adicciones. Para el primer caso, al ser la condición de estudiantes lo que el Estado requería, no hubo valoración de la territorialidad al momento de estructurar e implementar la política. Se interpelaba a jóvenes en tanto estudiantes. No ocurrió lo mismo con aquellos dispositivos que se crearon para población juvenil vulnerable o excluida, cuya institucionalidad fue implementada específicamente para ciertos barrios de la ciudad.

En todos los casos los beneficios consistieron en transferencias no condicionadas, pero el acceso a los mismos giró en torno a la prueba de medios (a partir de la detección efectiva de una problemática por parte de los profesionales municipales) o la afiliación (ser estudiante o vivir en determinados barrios de la ciudad). Claramente el efecto generado sobre la definición de los jóvenes fue, por un lado, estigmatizante (jóvenes con problemas - jóvenes adictos) y por otro estratificante respecto a los jóvenes (jóvenes incluidos - jóvenes excluidos /jóvenes estudiantes - jóvenes vulnerables). 
Por todo lo expuesto se puede afirmar que el sistema de protección social y bienestar juvenil que se adoptó desde el municipio para abordar la cuestión juvenil fue uno de carácter híbrido, con elementos dominantes del sistema "Fragmentado Residual" y del "Fragmentado Estratificante", conteniendo rasgos protectivos y productivos en sus dispositivos y sin presencia ni pretensión de universalidad.

\subsection{El sistema de protección social y bienestar juvenil bibrido "fragmentado estratificante e integral" de Villa Maria (1999-2017)}

A partir del estudio de la trayectoria de las políticas juveniles locales en la ciudad de Villa María, el análisis aborda los periodos de gestión comprendidos entre 1999 y 2017 [8], con lo cual se estructuró el abordaje de la cuestión juvenil en dos etapas: la primera que va desde 1999 a 2015, abarcando tres gestiones del ex intendente Eduardo Acastello (1999-2003; 2007-2011; 2011-2015) y una gestión de Nora Bedano (2003-2007). Aquí se han identificado tres momentos por las cuales pasaron las políticas juveniles, el de la génesis de la política (1999-2003); el momento de la profundización e institucionalización de la misma (2003-2011); y un tercer momento de amesetamiento y retroceso de la política juvenil que va entre 2011 y 2015 , La segunda etapa, es coincidente con la asunción en la gestión del actual intendente Martín Gill (2015-2019). Cabe resaltar que todas las gestiones estuvieron identificadas con el peronismo, siendo el Partido Justicialista de Villa María la columna vertebral de las distintas alianzas electorales que llegaron al gobierno durante todos estos años.

\subsubsection{Principales caracteristicas del sistema de protección social y bienestar juvenil durante la primera etapa (1999-2015)}

Durante este periodo, se divisaron características en las que las dimensiones construidas asumieron rasgos de las tres tipologías diseñadas para definir el sistema de protección. No obstante, se considera que en esta primera etapa en la ciudad se consolidó un sistema de protección "fragmentado estratificante" con un enfoque productivista. En este sentido el eje inclusión-exclusión adoptado por la gestión a la hora de diseñar y estructurar la implementación de políticas hacia el sector juvenil fue uno de los factores centrales que llevaron a esta caracterización. De acuerdo a Reguillo Cruz (2000), son dos los tipos de actores juveniles que el Estado muchas veces interpela; los "incorporados" y los "alternativos o disidentes”. Los primeros despliegan sus prácticas en el ámbito escolar, laboral y el mundo del consumo cultural mientras los segundos son los no incorporados al esquema de la cultura dominante. En Villa María se estructuró una división funcional entre dependencias al interior de la gestión de acuerdo a este eje de abordaje y al tipo de población juvenil que se deseaba alcanzar. Por un lado, el trabajo con jóvenes estudiantes (los incluidos) y por otro, las políticas sociales a jóvenes excluidos (los disidentes). De allí que se observó como una fuerte debilidad, la inexistencia de un trabajo articulado e integrado de los diversos organismos en torno a lograr un abordaje transversal y con enfoque generacional. 
El OGJ, se estructuró como un dispositivo destinado a la promoción de la participación política y el acceso a la cultura, interpelando de manera casi exclusiva a jóvenes estudiantes, mayoritariamente del nivel secundario. Por otra parte, desde las políticas sociales, se apuntó al abordaje del conjunto de problemáticas de los jóvenes excluidos - vulnerables. Al inicio de la primera gestión, este grupo de jóvenes era interpelado desde una noción estigmatizante ya que la institucionalidad generada se pensaba como una herramienta focalizada al "rescate y contención" de ciertos jóvenes en riesgo social, poniendo al Estado en un rol protector de aquellos jóvenes que por voluntad propia habían errado el camino (idea de ciudadanía invertida). Poco a poco esta mirada moralizante y de control social fue asumiendo la noción de que las intervenciones estatales debían promocionar su inclusión al sistema (educativo-laboral) como un modo de garantizar y mejorar sus condiciones de reproducción social ya sea como estudiante o trabajador (idea de ciudadanía regulada).

A su vez, por las graves consecuencias de la crisis socioeconómica del año 2001, el Estado local fue interpelado por una nueva realidad, jóvenes en situación de calle y con alta vulnerabilidad social, pertenecientes a los sectores populares de la ciudad. Apareció entonces un conjunto de intervenciones que aspiraban a dotar de capacidades a la juventud, con formación, capacitaciones, entrenamiento laboral, micro emprendimientos cooperativos, talleres culturales y recreativos como un modo de desarrollar en esta etapa de transición a la vida adulta, recursos y capitales sociales para su inclusión socioeconómica y el mejoramiento de sus condiciones de vida.

En cuanto a las políticas del organismo específico, al convocar a jóvenes ya incluidos y no identificarlos con vulnerabilidades, pareciera haber operado una lógica que entendía que este tipo de jóvenes eran los que estaban en "mejores condiciones" de ejercer como un "ciudadano", por ende, la preocupación no estuvo centrada en sus condiciones de reproducción social, sino más bien en profundizar sobre sus capacidades en la realización del derecho social de su ciudadanía. De allí que se generaron distintos dispositivos, con un enfoque de derechos, que promovieron la formación política y ciudadana, la participación, el ejercicio de la reflexión crítica y la posibilidad de transmitirlo al resto de la sociedad de una manera estratégica y legible.

En ambas estrategias, salvo proyectos puntuales, sí se observó que el conjunto de transferencias no estaba condicionado por contraprestaciones de los beneficiarios, propia del denominado sistema de protección y bienestar juvenil "integrado", de corte universalista. No obstante, en casi la totalidad de las acciones, para el acceso a los beneficios se convocó a los jóvenes según su afiliación (ser vulnerable o ser estudiante), propios del sistema "estratificante". Todas las políticas implementadas fueron focalizadas. Las acciones sociales apuntaron más al trabajo específico con grupos pequeños de adolescentes vulnerables y prestando especial atención sobre los territorios en los que las propuestas intervenían (barrios de sectores populares) y entendiendo la focalización como un modo de lograr la viabilidad y efectividad de sus propuestas, reconociendo que con los recursos disponibles cualquier tipo de masificación pondría en riesgo la consecución de los objetivos.

Los programas hacia estudiantes, también fueron focalizados y a su vez, en muchos de sus dispositivos se disponía un cupo máximo de participantes, pero 
sí apuntaban a la masificación cuantitativa de la propuesta, más como un modo de darle visibilidad y relevancia al conjunto de acciones que como una instancia de universalización de la acción estatal, por ende, no era necesario pensar territorialmente las propuestas ya que no había una asociación entre la población objetivo y el territorio del que provenían sino más bien de su institución: la escuela secundaria. No obstante, pudo observarse para el segundo momento de la gestión, que la territorialidad cobró mayor sentido en cuanto a la búsqueda de salir del ámbito escolar como lugar de despliegue de las prácticas, profundizando aspectos de socialización, conocimiento e intervención sobre la realidad social y comunitaria de los jóvenes estudiantes.

También se detectó que paulatinamente, hasta llegar al momento de auge e institucionalización de las políticas (2003-2011), el municipio comenzó a desplegar mayores intervenciones sobre la cuestión juvenil, destinando más recursos, construyendo infraestructura específica para el desarrollo de los programas, complejizando el conjunto de estrategias para el abordaje de lo juvenil. Esto tuvo como consecuencia el despliegue de mayores entramados institucionales y el desarrollo de diversos de aspectos burocráticos para la puesta en marcha diaria de las acciones.

Por último, puede afirmarse a partir de todas estas características que uno de los principales roles asumido desde el Estado durante estos primeros quince años fue la búsqueda del empoderamiento de la población juvenil en cuanto sus capacidades, conocimientos, formación académica, laboral y cultural destinada centralmente a su incorporación al mundo de las relaciones sociales en tanto jóvenes estudiantes y trabajadores.

A continuación, se analiza el periodo 2015-2017, en la que se divisaron importantes cambios en la institucionalidad del abordaje de lo juvenil, lo cual permite afirmar que se asiste a una nueva etapa de la política juvenil y con repercusiones específicas en la estructuración del sistema de protección juvenil local.

\subsubsection{Caracterización del sistema durante la segunda etapa de gestión (2015-2017)}

A partir del conjunto de modificaciones en la estructura de la institucionalidad sobre la juventud y del conjunto de programas destinados al abordaje de la cuestión juvenil, puede afirmarse que existió un corrimiento del tradicional eje inclusión-exclusión para pensar las principales políticas y las dependencias a cargo de las mismas. El cierre del OGJ, con la consecuente distribución de dos de sus tradicionales líneas de acción para jóvenes estudiantes a las nuevas dependencias, sumado a la desaparición del resto de sus intervenciones hacia la comunidad estudiantil pareciera haber marcado un nuevo perfil al abordaje de la cuestión juvenil: la apuesta por dotar de mejores condiciones de vida a jóvenes de sectores populares, a través del logro de la acreditación de la educación básica obligatoria.

No obstante, se dio continuidad al conjunto de políticas sociales destinadas a la formación, entrenamiento laboral, emprendimientos productivos y talleres recreativos. Éstas continuaron estando focalizadas hacia jóvenes vulnerables mediante dispositivos que perseguían trabajar con poca cantidad de beneficiarios, con la intención de lograr resultados efectivos de acuerdo a la estrategia de abordaje diseñada. Como ya se planteó para la primera etapa, estas políticas 
sociales apuntaban a una inclusión productiva juvenil y por ende con un efecto estratificante de las juventudes, en tanto buscaban la promoción y desarrollo de capacidades de los jóvenes para su reproducción social en tanto sujetos excluidos. El Estado se enfocó en la integración educativa y al mercado laboral como mecanismo de inclusión social. En algunos casos los beneficiarios debían generar una prueba de media (la condición de "vulnerable") para acceder a la política, pero era más bien su condición de desocupado o pertenencia territorial lo que primó en la incorporación de beneficiarios a estos programas.

Se observaron a lo largo de todo el recorrido histórico de la gestión, una serie de tensiones e incluso contradicciones en torno a las miradas sobre el/la joven y los paradigmas de trabajo. La situación recurrente fue la convivencia de enfoques de la juventud como transición o ciclo de vida, de corte evolucionista-biologicista, con el enfoque de derechos con una mirada más heterogénea, constructivista y no negativizante. Sin embargo, a diferencia de la primera etapa, no aparecieron durante la última gestión abordajes desde el enfoque de control social y miradas moralizantes-estigmatizantes de las juventudes como un problema.

Con el nuevo lineamiento de la política educativa juvenil a nivel local, apostando a la acreditación y finalización de los estudios básicos, se observó que la misma no se asentaba tanto en un enfoque de la juventud y la educación como una mera etapa de preparación y formación para la vida adulta, sino más bien como un desafío desde el Estado local por brindar oportunidades y garantías del efectivo ejercicio del derecho a la educación, siendo la misma una herramienta que promueve la autonomía juvenil y la constitución de proyectos de vida juveniles en mejores condiciones. Se perseguía un empoderamiento de los sujetos para decidir sobre su propia vida, partiendo del supuesto que la inclusión y titulación de la educación básica, lograría ampliar y multiplicar las posibles trayectorias de vida de los sectores populares. Es decir, una política que ha erigido a la justicia como principio, siendo su objetivo la restitución y redistribución de derechos sociales (particularmente la educación) y aspirando que toda persona que habita Villa María logre la finalización de la educación básica, o sea, una universalización de la condición de ciudadanía.

Si bien el programa se focalizó en aquellas personas que no terminaron el nivel medio, la política en sí mismo aspira a una universalización de la llegada al conjunto de jóvenes en dicha situación, desplegando una infraestructura institucional compleja y de corte más bien posburocrático para incorporar cada vez más personas al programa (para 2018 ya había mil personas inscriptas). En este sentido, el sistema institucional construido lejos de replicar el sistema educativo formal de la provincia de Córdoba ha realizado un entramado flexible, adaptado a las necesidades de la población beneficiaria, con un equipo técnico diverso e interdisciplinario para contener y abordar las múltiples realidades de los estudiantes. A su vez, implicó una misión de universalización de la condición de egresado de nivel medio en todas las y los jóvenes y en lo posible adultos, apostando a consolidar a Villa María como Ciudad del Aprendizaje.

Aunque muchos de las y los estudiantes provienen de los barrios de los sectores populares y la mayoría de los centros de formación se encuentran en estos lugares, la política se implementó en toda la ciudad, con centros en barrios de sectores medios y altos, con lo cual la territorialización de la política cobró mucho menos significancia, entendiendo que al abandono escolar y la ausencia de finalización 
del secundario atraviesa a todas las clases sociales, propia de modelos integrados de "inclusión universal". El programa no apuntó al joven pobre, sino al que no logró la finalización de sus estudios, sin importar su condición de clase o lugar de residencia.

Finalmente, a partir de las continuidades y modificaciones de la política juvenil implementadas por la nueva gestión, se puede afirmar que el sistema de protección social y bienestar juvenil es de carácter "híbrido", en un continuo entre el sistema "fragmentado estratificante" y el "integrado", profundizando su intervención en la cuestión juvenil sobre las trayectorias educativas de las juventudes villamarienses, sentando como base de su modelo de desarrollo social, la universalización de la educación básica. A continuación, en el Cuadro 2, se resumen las principales características que asumió el sistema municipal.

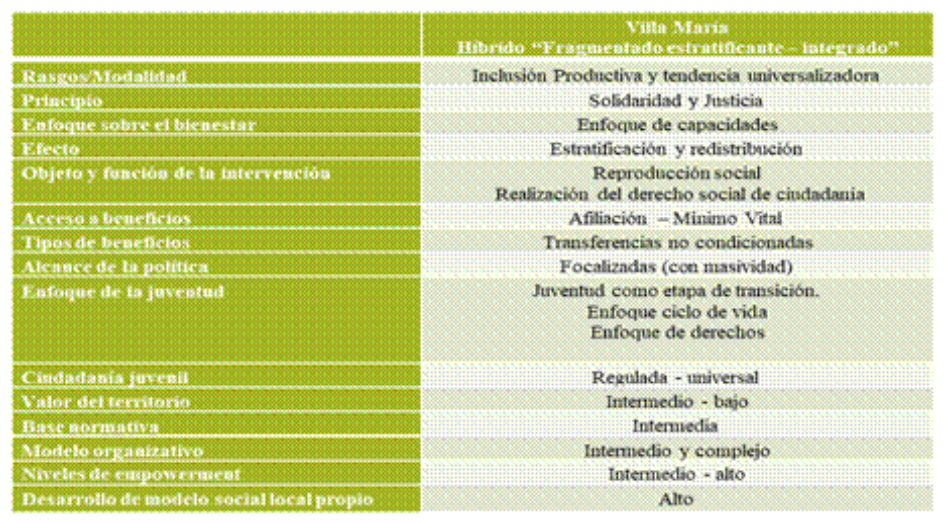

Cuadro 2.

Resumen de principales características del sistema de protección social y bienestar juvenil de Villa María (1999-2017) Elaboración propia en base a categorías de Donati y Lucas (1987); Fleury (2000); y Roca, Golbert y Lanari (2012), Brugué y Gomá (1998), Krauskopf (2000) y Rodríguez (2000 y 2003).

\section{Incidencia de los factores en la conformación de los sistemas de protección social y bienestar juvenil}

A los efectos de un análisis cualitativo y comparativo de los casos seleccionados, en este apartado se presentan en términos generales las características que asumieron las variables establecidas como marco explicativo en los municipios de Villa María y Jesús María exponiendo los principales hallazgos de las relaciones de las variables y su impacto en la conformación de los tipos híbridos de sistemas de protección social y bienestar juvenil que se definieron para cada caso en el apartado anterior.

5.1 El vínculo de los proyectos de gobierno con los sistemas de protección social y bienestar juvenil

Más allá de los distintos ejes que cada gestión resaltó o reseñó como centrales en cada periodo de gestión, se observa que los principales lineamientos asignados al proyecto de gobierno efectivamente intervinieron en las nociones sobre el rol de la intervención estatal en la cuestión social y específicamente, la juvenil. 
Por una cuestión de extensión, se presenta de manera esquemática y resumida en el Cuadro 3 las principales características que sumieron los proyectos de gobierno en ambos municipios para todo el periodo analizado.

\begin{tabular}{|c|c|c|}
\hline & Villa María & Jesús María \\
\hline Autodenominación & "Peronista-Nacional y Popular" & "Progresista-socialdemócrata" \\
\hline Misión de gobierno & $\begin{array}{l}\text { Refundar el Estado local / Construir una } \\
\text { Comunidad Organizada }\end{array}$ & Modernizar el Estado \\
\hline $\begin{array}{l}\text { Rol del estado en la } \\
\text { dinámica local }\end{array}$ & $\begin{array}{l}\text { Promotory coordinador de una democracia } \\
\text { protagónica. } \\
\text { Principal proveedor de servicios públicos. }\end{array}$ & $\begin{array}{l}\text { Promotor del desarrollo } \\
\text { Principal proveedor de servicios públicos }\end{array}$ \\
\hline $\begin{array}{l}\text { Rol del Estado en la cuestión } \\
\text { social }\end{array}$ & $\begin{array}{l}\text { Construir condiciones materiales y espirituales de } \\
\text { vida para la inclusión social. Promoción del } \\
\text { derecho de ciudadanía }\end{array}$ & $\begin{array}{l}\text { Promover la igualdad de oportunidades y el } \\
\text { derecho de ciudadanía. }\end{array}$ \\
\hline $\begin{array}{l}\text { Modelo de desarrollo de } \\
\text { ciudad }\end{array}$ & $\begin{array}{l}\text { Movilidad social ascendente, equidad, justicia } \\
\text { social. El ser humano como eje de la política }\end{array}$ & $\begin{array}{l}\text { Desarrollo armónico con paz social. } \\
\text { El ser humano como centro de la política. }\end{array}$ \\
\hline Tipo de Estado & Relacional-Interventor - Descentralizado & Relacional-Promotor \\
\hline Ejes de gestión & $\begin{array}{l}\text { Eficiencia, eficacia, participación ciudadana, } \\
\text { gestión asociada, equilibrio fiscal, sustentabilidad, } \\
\text { trabajo y desarrollo productivo, modernización de } \\
\text { la organización estatal, inclusión e integración } \\
\text { social, ciudad segura. }\end{array}$ & $\begin{array}{l}\text { Eficiencia, eficacia, transparencia, participación } \\
\text { ciudadana, equilibrio fiscal, , inclusión social, } \\
\text { modernización de la organización estatal, } \\
\text { sustentabilidad, ciudad segura. }\end{array}$ \\
\hline $\begin{array}{l}\text { Tipo de Proyecto de } \\
\text { Gobiemo }\end{array}$ & Posneoliberal & Posneoliberal \\
\hline
\end{tabular}

\section{Cuadro 3.}

Características de Proyectos de Gobierno en Villa María y Jesús María elaboración propia en base a documentos institucionales y entrevistas a funcionarios de Villa María y Jesús María.

En Villa María la apuesta del proyecto por lo educativo y la inclusión social se tradujo en la conformación de una institucionalidad estratificante ordenada alrededor del eje joven incluido-joven excluido. De esta manera, la prioridad de promover la educación formal en niños y jóvenes, alrededor de instalar a la localidad como "ciudad del conocimiento" (primera gestión de Acastello) o "ciudad del aprendizaje" (gestión de Gill) configuró los principales lineamientos que la política juvenil adquirió en esos momentos, influyendo en el arreglo institucional que desplegaron las dependencias creadas a tales fines. A su vez, en la apuesta del proyecto por mejorar las condiciones de vida de la población mediante la gestión de la cuestión social, estuvo atravesada por la idea de resolver los principales riesgos sociales mediante el desarrollo de capacidades, evitando una óptica asistencial. Como consecuencia la política social juvenil se caracterizó por apostar al desarrollo de la autonomía en la definición de los proyectos de vida de los jóvenes. Para ello la capacitación, el entrenamiento laboral, lo cultural y deportivo y, el desarrollo de microemprendimientos fueron mecanismos centrales. No obstante, y a pesar del posicionamiento político posneoliberal o progresista del proyecto, el sistema de protección asumió un carácter fragmentado, detectando tensiones y contradicciones en torno a la comprensión en el abordaje de la cuestión juvenil, el enfoque de las políticas y el tipo de institucionalidad desarrollada.

Por su parte, las gestiones de Jesús María establecieron como prioridades en sus ejes de gestión la transparencia, inversión pública, eficiencia, participación e inclusión social, cuestiones que impactaron sobre el sentido general de la política juvenil en los momentos que atravesó la misma. Concretamente, por un lado, el eje de la participación política fue lo que determinó la principal finalidad del organismo juvenil creado. Por otro lado, la preocupación por los riesgos sociales juveniles en torno a problemáticas como las adicciones fue el otro lineamiento que determinó el segundo aspecto central de la política juvenil en el periodo. La 
mayor o menor relevancia del abordaje estatal de la cuestión juvenil local también estuvo determinada por los ejes del proyecto político, como se observó con lo sucedido en las dos gestiones de Frizza y su decisión política de tener o no a la juventud en su agenda de gobierno, impactando sobre la performance de la política juvenil y el desarrollo de institucionalidad.

$\mathrm{Al}$ igual que en Villa María el sistema desarrollado fue fragmentado, pero se diferenció en la modalidad que adquirió el mismo. El municipio tuvo un rol estatal sobre la cuestión juvenil mucho más acotado, con un menor despliegue institucional y no exento de tensiones y contradicciones en los enfoques de la política, viéndose reflejado en la fragmentación de tipo residual y estratificante del sistema.

De acuerdo al esquema analítico planteado, los lineamientos de un proyecto de gobierno no permiten explicar de manera acabada e integral la forma y contenido que adquieren los sistemas de protección social y bienestar juvenil. En términos de los casos analizados surge la siguiente pregunta: ¿̇por qué teniendo ambos proyectos de gobiernos un carácter posneoliberal, en Jesús María se estructuró un sistema híbrido entre el modelo fragmentado residual y estratificante, mientras que en Villa María fue un sistema híbrido que se ubicó entre una fragmentación estratificante y el impulso de un modelo integrado?

Dadas las similitudes de la variable proyecto de gobierno en ambos casos estudiados, la misma no es suficiente para dar respuesta a este interrogante. No obstante, podría pensarse que, si bien las diferencias en el proyecto de gobierno no son sustanciales para dar cuenta de las particularidades que asumieron cada uno de los sistemas de protección, estas diferencias podrían explicarse por los distintos compromisos efectivos con dicho proyecto en cada municipio. Tal compromiso puede establecerse a partir de las capacidades estatales generadas para llevarlo adelante. El próximo apartado se ocupa de esta cuestión.

\subsubsection{Las injerencias de las capacidades estatales en la estructuración de los sistemas de protección social y bienestar juvenil}

Se considera que, dentro del conjunto de dimensiones analizadas sobre las capacidades estatales, el perfil del personal seleccionado jugó un papel central en el diseño de las políticas juveniles, mediante su injerencia en la determinación de sus elementos, los sentidos de la misma y los modelos de abordaje que sustentaban la intervención. Todos estos son factores fundamentales en la estructuración de los sistemas de protección social y bienestar juvenil.

Los equipos técnicos conformados se transformaron en los intérpretes de los lineamientos políticos generales dispuestos por el proyecto de gobierno, e incluso cuando de éste no surgieron objetivos claros y concretos sobre el abordaje de lo juvenil, el personal de las dependencias fue quien los configuró. Puede afirmarse que es principalmente el personal a cargo de la política los que construyen las producciones estatales sobre la cuestión juvenil y las juventudes como causa pública a nivel local en ambos municipios.

En la medida que el abordaje de lo juvenil se fue institucionalizando a través de distintas dependencias, según el tema específico a abordar, en cada una de ellas operaron distintos criterios en la selección de los perfiles del personal y fueron los profesionales a cargo de la política los que estructuraron a su vez el perfil de las 
unidades estatales dirigidas a la cuestión juvenil. Como afirmaba una funcionaria de Villa María:

La discusión del concepto de la política [juvenil] (...) tiene que ver con las trayectorias de cada quien. Si yo acá estoy trabajando con sociólogos, y además en mi equipo tengo todos pedagogos ¿no?, en la escuela granja la mayoría son de ciencias políticas... y ahí sesgás, sesgás la mirada, entonces cada quien va mirando, entonces el sentido en la cotidianidad de la gestión también te lo da tu trayectoria, y cuando vos te sentas, o presentas, cuando nos ha pasado en algunas reuniones intersecretaría, cada quien piensa y mira desde su propia trayectoria (Entrevista 24).

En este sentido mientras más dividido y segmentado se encuentre el sentido y la institucionalización del abordaje de la cuestión juvenil, más diferenciado sea el ámbito de intervención de cada dependencia, y más diversos sean los perfiles profesionales, sin existir mecanismos institucionales que le den transversalidad, integralidad y coherencia interna a la política juvenil, más fragmentada será la conformación de los sistemas de protección social y bienestar juvenil.

Desde el análisis en profundidad pudo establecerse cómo se conjugaron estos factores al momento de fragmentar sus sistemas de protección. Así fue que en Jesús María las definiciones sobre la juventud y la cuestión juvenil perfilaron una política centrada en un organismo específico que promovió la participación política de jóvenes estudiantes entre 15 y 20 años de edad combinándose con intervenciones más sanitaristas para la prevención de adicciones de jóvenes de sectores populares (desde otra dependencia) y acciones de prevención indirecta sobre consumos problemáticos en estudiantes secundarios. El perfil del personal seleccionado y la ausencia de una "línea clara" de las autoridades en torno a la política juvenil habilitó que cada unidad administrativa tuviera completa autonomía para definir los enfoques y las características que las políticas asumieron. La escasez de recursos humanos y económicos ancló a las intervenciones en pequeños dispositivos, que para garantizar la incorporación de beneficiarios/as recurrió al "público cautivo" de estudiantes de las escuelas de nivel medio. Además, en los momentos en que predominó una lógica políticaelectoral en la selección del personal se observó que los conflictos que se desataron debilitaron el desarrollo de las políticas y la institucionalidad juvenil. A partir de esta estructuración el sistema de protección juvenil de la ciudad se fragmentó entre las modalidades "residual" y "estratificante".

En Villa María la definición de la cuestión juvenil fue más amplia con lo cual el rol de la intervención del Estado local se diversificó y tuvo un mayor despliegue que en Jesús María. Éste se estructuró en torno a las dos preocupaciones centrales de la condición del joven en la ciudad, es decir el carácter incluido/excluido del mismo, segmentando las intervenciones en dos dependencias. Por un lado, un organismo específico que abordó temáticas educativas y de promoción de la participación política de jóvenes estudiantes de nivel medio, y en menor medida, de nivel superior. Por otro lado, la política social juvenil bajo la órbita de las áreas sociales que estructuraron sus intervenciones alrededor de las problemáticas socio-económicas de las y los adolescentes de sectores populares. En consecuencia, el sistema se configuró bajo una modalidad fragmentada estratificante. En la última etapa de gestión, con el cierre del organismo y al establecer la terminalidad como una línea central de acción atravesando al conjunto de las políticas juveniles, se incorporaron elementos propios de un 
sistema de protección más integrado. Las políticas fueron acompañadas por una importante dotación de recursos, que fueron fundamentales para el despliegue de las intervenciones estatales, sobre todo las políticas sociales juveniles y de terminalidad educativa que se consignaron como líneas centrales y que por sus objetivos y alcance implicaron un importante desarrollo de institucionalidad.

Cabe resaltar que, en ambos gobiernos locales, las características generales que asumieron los diferentes aspectos de sus capacidades estatales contribuyeron y reforzaron, en distinta medida según el caso, la lógica fragmentada de los sistemas. De manera breve se resumen las mismas:

- La utilización de criterios políticos-electorales o políticos-técnicos al momento de conformar los equipos de profesionales a cargo de las dependencias afectaron sobre la estabilidad de la política y el desarrollo de institucionalidad. Principalmente para el caso de Jesús María, se observó que la preeminencia del primero sobre el segundo impactó negativamente sobre las políticas.

- La ausencia de una perspectiva generacional y el establecimiento de objetivos claros respecto a la cuestión juvenil y el modo de su abordaje desde las "primeras líneas de funcionarios" habilitó la "libertad de acción" de cada dependencia. Esto aumentó la fragmentación de las intervenciones y atentó contra la coherencia interna de la intervención estatal.

- Los enfoques y perspectivas con que imprimieron el abordaje de la cuestión juvenil se ajustó según el perfil profesional del personal seleccionado, sin un lineamiento común que ordenara a los mismos. En consecuencia, se multiplicó la coexistencia de distintos paradigmas y sentidos de la política, generando tensiones y contradicciones sobre el modo de concebir al joven y la juventud.

- La falta de disponibilidad de recursos (humanos y financieros) no sólo fue un indicador de la relevancia que adquirió el tema en la agenda política, sino que la escasez de los mismos tendió a promover la implementación de pequeños programas focalizados, lo que fragmentó aún más los sistemas. En el caso de Jesús María su impacto fue notorio.

- La ausencia de autoridad jurídica de las dependencias para administrar las partidas de sus propios presupuestos atentó contra el fortalecimiento de las políticas juveniles. En varios momentos esto generó que la dotación de recursos económicos dependiera de la capacidad política de gestión de los referentes a cargo de la política juvenil y no de la planificación estipulada sobre el abordaje de lo juvenil.

- Las condiciones laborales de los equipos técnicos afectaron sobre la estabilidad de las políticas y la robustez del sistema. A menores condiciones, mayores fueron los problemas en torno al desarrollo de institucionalidad y despliegue de la política diseñada.

- La expresión de voluntad o intención política de los proyectos de gobierno por abordar integralmente la cuestión juvenil no fue garantía suficiente para que esto ocurra. Se necesita un desarrollo institucional que promueva y canalice su concreción. 
Las similitudes en las capacidades de ambos casos dan cuenta de lo que ambos sistemas comparten, su carácter fragmentado. Es decir, un proyecto posneoliberal que genera capacidades como las descriptas, lleva a un sistema fragmentado. Sin embargo, la dotación de recursos es un elemento que da cuenta de lo que principalmente separa a ambos sistemas, mientras que el resto de las características administrativas indicaría lo que los une. En términos presupuestarios y dotación de recursos, Villa María muestra mucho mayor compromiso que Jesús María.

Las diferencias de los presupuestos comprometidos para las políticas juveniles y su representación en el presupuesto de políticas sociales y el presupuesto general son notables. Respecto a la cuestión juvenil, esto se tradujo en una importante batería de políticas sociales que intentaron abordar múltiples situaciones problemáticas que las juventudes de sectores populares atravesaban. Estas intervenciones territoriales a lo largo del tiempo fueron generando su propia institucionalidad, que necesitaron para su robustez y permanencia en el tiempo, la asignación de importantes recursos. A su vez, en la segunda etapa, el objetivo de hacer de la localidad una "Ciudad del Aprendizaje" se plasmó en el diseño e implementación de un ambicioso y masivo plan de terminalidad educativa para jóvenes. Este desafío se efectivizó mediante un complejo despliegue de herramientas y mecanismos institucionales que lograron incorporar en la actualidad a más de mil beneficiarios/as.

\subsection{Los actores de la dinámica política local en el abordaje de la cuestión juvenil}

Respecto a los factores que influyen sobre la determinación del tipo de sistema de protección social y bienestar juvenil, además de los proyectos de gobierno, se consideró también los procesos de inclusión/exclusión de actores sociales que influyeran en la constitución de la agenda social de gobierno y la gestión de las políticas. Para ello, se debía reconocer quiénes y en qué momento se interesaron por la cuestión juvenil a nivel local y con qué recursos económicos, políticos, organizacionales y simbólicos contaron en la disputa con otros sectores al momento de la conformación de la agenda gubernamental (Repetto, 2002).

A partir del análisis realizado para el municipio de Jesús María y Villa María se ha establecido la ausencia de importantes actores extraestatales generando presión e invirtiendo recursos para influenciar sobre los proyectos de gobierno o tener injerencia directa respecto al abordaje de la cuestión juvenil local o impactar sobre el diseño e implementación de las políticas juveniles. En las entrevistas, los propios agentes estatales, funcionarios/as (de primera a tercera línea jerárquica) e intendentes/as, no otorgaron relevancia a ningún actor con capacidad de intervenir en la dinámica de la gestión respecto a lo juvenil. Además, no se divisó en el diseño de las políticas, el despliegue de articulaciones en conjunto (Estadoactores sociales) de algún programa o proyecto y fueron pocos los casos en la que, al menos en la etapa de implementación, se incluyeron actores del territorio para la ejecución de algunas acciones. ${ }^{9}$

No obstante, siguiendo con otro aspecto del marco teórico propuesto respecto a la existencia de actores al interior del subsistema político estatal se relevó la existencia de dos actores que tuvieron distintos tipos de influencia según el momento de la política. Por un lado, a partir de las negociaciones políticas que 
los proyectos de gobierno realizaron para la conformación de las coaliciones gobernantes, la coordinación de los organismos juveniles específicos en ambos municipios funcionó en determinadas épocas como "prenda de intercambio y negociación” con organizaciones políticas partidarias que formaron parte de la alianza de gestión.

En este sentido, para el caso de Jesús María, y en menor medida en Villa María, se visualizaron instancias de negociación entre los intendentes y ciertas organizaciones políticas pertenecientes a la alianza de gobierno, para acordar la distribución de cargos en la estructura orgánica municipal a nivel general y en las dependencias a cargo de lo juvenil en particular. Además, existieron lobbies políticos al interior de la gestión para que las y los funcionarios y miembros del equipo que no militaban en alguna organización de la coalición gobernante tuvieran una incorporación paulatina como afiliados/as, miembros activos o dirigentes en la estructura del partido de gobierno.

Como contrapartida, en los momentos donde primó un criterio más técnico que político-electoral en la selección de los perfiles de los funcionarios en los OGJ, se observó un esfuerzo de las y los nuevos coordinadores (frente a la sociedad y al propio gobierno) por despegar políticamente a la dependencia, y sus jóvenes beneficiarios/as, con las estructuras partidarias y los momentos electorales. Es decir, el área y las juventudes alcanzadas no serían un apéndice o brazo juvenil institucionalizado del partido.

Por otra parte, sí se divisó la importante injerencia que asumieron los perfiles profesionales de los equipos a cargo de la política al interior de las dependencias propiciaron que estos se conformaran como "coaliciones promotoras" del abordaje de lo juvenil impulsando su "sistemas de creencias" respecto a la cuestión al interior de los organismos y en la gestión en general.

Desde la teoría de las coaliciones promotoras se consideran a las ideas y las definiciones de un problema como variables explicativas de la estabilidad o cambio de políticas (o modificación del núcleo de la misma). Éstas son analizadas empíricamente a través de dos instrumentos: el sistema de creencias y el aprendizaje. ${ }^{10}$

En los dos municipios estudiados los equipos técnicos terminaron conformándose como coaliciones promotoras que promovieron su propio sistema de creencias en la conformación del núcleo de las políticas. Las principales modificaciones al interior de las políticas juveniles coincidieron con los momentos en que las y los funcionarios y miembros de los equipos técnicos eran reemplazados por nuevos profesionales. También se detectaron influencias externas que impulsaron "aprendizajes" sobre el quehacer en la política juvenil.

Se resalta también que a lo largo de los distintos momentos de la política estas coaliciones funcionaron de manera coordinada ante tensiones, conflictividades, intentos de injerencia, recelos y competencias de otras dependencias, como un modo de "proteger y cuidar" su propia influencia sobre el núcleo de las políticas que desplegaban.

Estas coaliciones promotoras se consolidaron como "comunidades epistémicas" (Haas, 1992) en torno al abordaje de la cuestión juvenil. Por comunidad epistémica se entiende a una "red de profesionales con una expertise y una competencia en un tema determinado, reconocidas estas por otros y que reclaman autoridad sobre un conocimiento que es relevante para la formulación 
de políticas al interior de ese tema o campo" (Haas, 1992, en Gómez Lee, 2012 p. 14). En este sentido, dichas comunidades poseen un conjunto compartido de creencias en base a principios, los cuales dotan de racionalidad a la intervención que se realice sobre ella; hay creencias causales compartidas que marcan el vínculo entre las políticas posibles y los resultados esperados; poseen nociones compartidas de validez, desde las cuales dan entidad al conocimiento sobre el tema y; constituye una empresa común con la convicción de dar respuesta a la problemática que se aborda (Haas, 1992). El conjunto de cosmovisiones, representaciones, concepciones de las juventudes; definiciones del rol estatal sobre la cuestión juvenil y las acciones diseñadas a los fines de abordar a las mismas dieron cuenta del carácter de actor que asumieron estas comunidades epistémicas de profesionales estatales durante los periodos temporales analizados.

\section{Reflexiones finales}

Luego del análisis realizado sobre la dinámica asumida por las variables propuestas para explicar la estructuración de los sistemas de protección social y bienestar juvenil en los municipios seleccionados, se llega a la conclusión de que la decisión de la intervención del Estado local sobre la cuestión social, y específicamente la cuestión juvenil y los lineamientos generales que los sistemas adquirieron, estuvieron condicionados por las características posneoliberales que asumieron los proyectos de gobierno locales.

Ahora bien, la traducción de las definiciones políticas-ideológicas de los proyectos de gobierno sobre el quehacer estatal en la cuestión juvenil estuvo principalmente mediada en la práctica concreta, por el efectivo despliegue de las capacidades estatales que se abocaron al diseño y ejecución de la política juvenil. Las capacidades estatales tienen una incidencia respecto del carácter fragmentado o no de los sistemas. A su vez, las colaciones promotoras conformadas al interior de las unidades administrativas tuvieron un importante impacto en el abordaje de la cuestión juvenil. Éstas definieron en gran parte aspectos centrales como: la definición de sobre las juventudes y la cuestión juvenil que asumió el Estado, los principios y efectos de la política, los enfoques sobre el bienestar y la juventud, el objeto y función de la intervención, los beneficios otorgados y condicionalidades para acceder al mismo y el tipo de ciudadanía juvenil que promocionan las intervenciones. Mientras más adultocéntricos fueron los sistemas de creencias desarrollados por las comunidades epistémicas al interior de las dependencias en torno a lo juvenil, más residuales y estratificantes fueron las políticas implementadas

No obstante, la perdurabilidad y permanencia de estas comunidades epistémicas de profesionales al interior del Estado estuvo influenciada por las prioridades de agenda y decisiones políticas-electorales que el proyecto de gobierno realizó a lo largo de sus gestiones. Mientras mayores fueron las injerencias de lo político-electoral sobre el diseño institucional y su dotación de recursos (humanos y financieros) mayor fue la inestabilidad de la institucionalidad juvenil conformada, afectando (positiva o negativamente) sobre la continuidad de los equipos técnicos y las políticas que éstos llevaban adelante. A su vez, la ausencia de actores extraestatales que presionen o ejerzan influencia sobre la cuestión juvenil local, dotó de mayor discrecionalidad al 
Estado (desde los proyectos de gobierno y las coaliciones promotoras internas) para determinar el sentido del sistema de protección social y bienestar juvenil en cada municipio.

En síntesis, el acento sobre los factores explicativos de los sistemas de protección estuvo centrado en las características que asume un proyecto de gobierno y su compromiso en la implementación a partir de las capacidades estatales efectivamente desplegadas como resultado de las disputas que las coaliciones promotoras de las dependencias a cargo de la cuestión juvenil realicen al interior de la gestión. En este sentido se afirma que un sistema de protección social y bienestar juvenil tenderá a ser integrado en la medida que sea llevado adelante por un proyecto de gobierno posneoliberal que efectivamente se compromete en el despliegue de capacidades estatales y cuente con coaliciones promotoras que promuevan un enfoque integral en el núcleo de la política e instalen perspectiva generacional al conjunto de la gestión. Mientras menor sea el compromiso en el desarrollo de este tipo de capacidades y más disímiles y débiles sean las coaliciones promotoras sobre la cuestión juvenil al interior de un gobierno, mayor será el grado de fragmentación que adquiera el sistema.

\section{Referencias}

Acuña, C. (2005). Notas sobre la metodología para comprender (y mejorar) la lógica político-institucional de las estrategias de reducción de la pobreza en América Latina. Documento de trabajo, 10. Buenos Aires: Fundación PENT.

Acuña, C. (2013). Cuánto importan las instituciones. Gobierno, Estado y actores en la politica argentina. Buenos Aires: Siglo XXI.

Alegre, P. (2008). "Los giros a la izquierda en el Cono Sur: gobiernos progresistas y alternativas de desarrollo en perspectiva comparada". Informe final del concurso: Gobiernos progresistas en la era neoliberal: estructuras de poder y concepciones sobre el desarrollo en América Latina y el Caribe. Montevideo: CLACSO.

Balardini, S. (1999). "Políticas de juventud: conceptos y la experiencia argentina". Última Década 10(3). Viña del Mar: CIPDA.

Bobbio, N. (1995). Izquierda y derecha, Razones y significados de una distinción política, Madrid, España: Taurus.

Brugué, Q. y Gomá, R. (1998). Gobiernos locales y politicas públicas: bienestar social, promoción económica y territorio. Barcelona: Editorial Ariel.

Cecchini, S., Filgueira, F. y Robles, C. (2014). Sistemas de protección social en América Latina y el Caribe: una perspectiva comparada, Serie Políticas Sociales, № 202, Santiago de Chile: CEPAL.

Centro Latinoamericano de Administración para el Desarrollo - CLAD. (1997). Una nueva gestión pública para América Latina. Buenos Aires: CLAD.

Donati, P. y Lucas, A. (1987). La política social en el Estado de Bienestar: el desafío de los sistemas complejos. Reis, 37, 57-68.

Fleury, S. (2000). "Reforma del Estado: diseño y gerencia de políticas y programas sociales". Banco Interamericano de Desarrollo, Instituto Interamericano para el Desarrollo Social (INDES). Washington, DC.

Giordano, V. (2014). ¿Qué hay de nuevo en las «nuevas derechas»? Nueva sociedad, (254), 49 . 
Gómez Lee, M. (2012). El marco de las coaliciones de causa-Advocacy Coalition Framework. Revista Opera, (12), 11-30.

Haas, P. M. (1992). Introduction: epistemic communities and international policy coordination. International organization, 46(1), 1-35.

Krauskopf, D. (2000). “Dimensiones críticas en la participación política de los jóvenes” en Balardini, S (comp.) La participación socialy politica de los jóvenes en el horizonte del nuevo siglo. Ciudad Autónoma de Buenos Aires: CLACSO.

Laham, M. (2010). El Modelo Biopsicosocial. Disponible en http:// www.psicologiadelasalud. com.ar. Inédito.

Lievesley, G., \& Ludlam, S. (2009). Introduction: A “Pink Tide”, en: Lievesley, G.; Ludlam, S. (eds.), Reclaiming Latin America: experiments in radical social democracy, London: Zed Books, pp. 1-18.

Nazareno, M. (2009). Hacer la diferencia. Nueva izquierda, coaliciones y politica de la política económica. La Argentina en el contexto latinoamericano, 2003-2007, Tesis doctoral, Facultad Latinoamericana de Ciencias Sociales, Argentina.

Oszlak, O. (2004). "Privatización y capacidad de regulación estatal: una aproximación teórico-metodológica”, en Politica y Gestión Pública, Buenos Aires: FCE/CLAD.

Ramirez Gallegos, F. (2012a). "Crisis neoliberal y reconfiguraciones estatales: Ecuador y la heterodoxia sudamericana”, en Revista Linea Sur, Vol. 1, Issue 2, pp. 84-104.

Reguillo Cruz, R. (2000). Emergencia de culturas juveniles. Estrategias del desencanto. Buenos Aires: Norma.

Repetto, F. (2002). Los actores de la politica social, mimeo, Washington D. C.

Roca, E.; Golbert, L.; Lanari M.E. (2012). ¿Piso o sistema integrado de protección social? Una mirada desde la experiencia argentina. Buenos Aires, Ed. Ministerio de Trabajo, Empleo y Seguridad Social.

Rodríguez, E. (2003). Políticas públicas de juventud en América Latina: de la construcción de espacios específicos, al desarrollo de una perspectiva generacional. Revista Latinoamericana de Ciencias Sociales, Niñez y Juventud, 1(2), 15-43.

Rodríguez, E. (2000). "Juventud y políticas públicas en América Latina: experiencias y desafíos desde la gestión institucional”, Última Década 8(13), 35-58. Viña del Mar: CIDPA.

Sader, E. (2008). Posneoliberalismo en América Latina. Buenos Aires: Clacso.

Scagliola, M. (2018). "La transición a la adultez. Apuntes para un balance de tres décadas de políticas de juventud en Uruguay”. En Beretta, D., Laredo, F. Nuñez, P. y Vommaro, P. (comp.) Políticas de juventudes y participación politica. Rosario, Argentina: Universidad Nacional de Rosario, pp. 273-292.

Stokes, S. (2008). Globalization and the Left in Latin America. Yale University, Department of Political Science.

Stoessel, S. (2014). Giro a la izquierda en la América Latina del siglo XXI: Revisitando los debates académicos. Polis (Santiago), 13(39), 123-149.

Viguera, A. (2000). Estado, empresarios y reformas económicas: en busca de una perspectiva analítica integradora. Zona abierta, (90-91), 161-203. Madrid.

\section{Notas}

1 Un desarrollo acabado del esquema analítico y metodológico de la investigación en la que se inscribe este trabajo puede leerse en Autor (2020a). 
2 El plano de la contraposición es fundamental ya que como afirma Norberto Bobbio "lo que es de izquierda lo es con respecto a lo que es de derecha" (Bobbio citado en Giordano, 2014, p. 48).

3 “...el "nuevo golpismo” está encabezado más abiertamente por civiles y cuenta con el apoyo tácito (pasivo) o la complicidad explícita (activa) de las Fuerzas Armadas, pretende violar la constitución del Estado con una violencia menos ostensible, intenta preservar una semblanza institucional mínima (por ejemplo, con el Congreso en funcionamiento y/o la Corte Suprema temporalmente intacta), no siempre involucra a una gran potencia (por ejemplo, Estados Unidos) y aspira más a resolver un impasse social o político potencialmente ruinoso que a fundar un orden novedoso" (Tokatlian, Juan Gabriel. “Neogolpismo”. Página/12 Web. Buenos Aires, 13/07/2009).

4 En Autor (2019) puede observarse un análisis en profundidad de este caso en particular.

5 Paradójicamente el programa se llamó “Jóvenes ciudadanos". No obstante, desde el programa juvenil el principal objetivo era prevenir a los jóvenes de los factores de riesgo que conlleva a las adicciones. Para ello, era necesario tratarlos clínicamente y profundizar en diversos aspectos para el desarrollo de las capacidades. Para abordar conjuntamente estos problemas adoptaron como enfoque el modelo Biopsicosocial (Laham, 2010) determinando una serie de lineamientos: la asistencia para los usuarios de sustancias, la prevención específica para evitar o reducir el consumo de sustancias, y la prevención inespecífica que, de manera global, buscaba abordar las necesidades y motivaciones del consumo.

6 En este primer momento se implementaron nueve líneas de acción, los programas y proyectos de asistencia y prevención directa se destinaron a la atención y prevención de riesgos de jóvenes "adictos" de sectores populares. Por otra parte, el resto de los programas de prevención indirecta estuvieron diseñados para fomentar la participación y formación ciudadana.

7 En este contexto, el organismo decidió sostener algunos programas de la gestión anterior, pero se resinificaron y modificaron sus objetivos y metodologías acorde al nuevo enfoque de abordaje. Además, desde el Área se crearon siete nuevas líneas de acción.

8 Un análisis pormenorizado de este caso de estudio puede verse en Autor (2020b)

9 Cabe preguntarse además, si la ausencia de actores extraestatales participando en la dinámica estatal no es también un reflejo de cierta contradicción entre los discursos gubernamentales y sus políticas: ambas gestiones municipales incluyeron en sus ejes de gobierno una fuerte incentivación de la participación ciudadana y juvenil en particular, poniendo el acento en la construcción de un nuevo Estado relacional, dialógico, haciendo parte a su comunidad de las decisiones del rumbo de las gestiones municipales. No obstante, en lo que respecta a la especificidad de esta investigación, no se observó un impulso desde los propios gobiernos locales en la creación de su institucionalidad con mecanismos y herramientas en la que las juventudes y organizaciones del campo juvenil tengan un rol protagónico en la construcción de los sistemas de protección social y bienestar juvenil.

10 Las dos hipótesis centrales de la teoría consisten en que los núcleos de las políticas no se modificarán significativamente hasta en tanto la coalición promotora que inició el programa se mantenga en el poder, salvo orden de jurisdicción de jerarquía superior. La segunda hipótesis consiste en que las perturbaciones externas al subsistema son causas necesarias, pero no suficientes para explicar un cambio en el núcleo de las políticas.

Sobre el autor

\section{Notas de autor}

Doctor en Política y Gobierno y Licenciado en Ciencia Política de la Facultad de Ciencia Política y Relaciones Internacionales de la Universidad Católica de Córdoba - UCC. Magister en Diseño y Gestión de Programas Sociales (FLACSO - Argentina). Becario Posdoctoral (CONICET) radicado en el CIECS (CONICET y UNC). Miembro del GT Infancias y Juventudes de CLACSO. Docente de grado en la Licenciatura en Ciencia Política de la Facultad de Ciencia Política y Relaciones Internacionales de la UCC. 